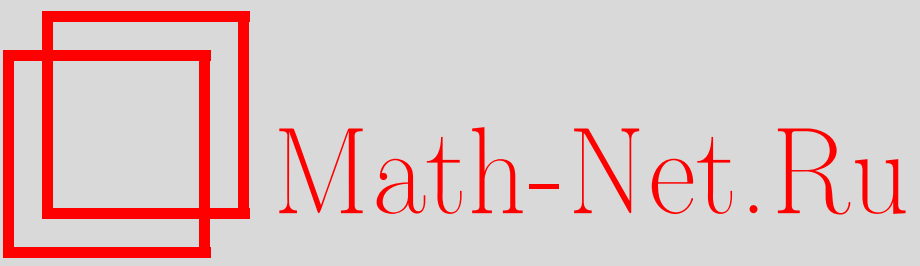

А. Л. Крылов, Г. А. Эль, Стохастическое описание конечнозонных потенциалов, УМH, 1999, том 54, выпуск 2, 175-176

DOI: https://doi.org/10.4213/rm140

Использование Общероссийского математического портала Math-Net.Ru подразумевает, что вы прочитали и согласны с пользовательским соглашением

http://www . mathnet.ru/rus/agreement

Параметры загрузки:

IP: 18.208 .226 .222

26 апреля 2023 г., 17:00:03 


\title{
СТОХАСТИЧЕСКОЕ ОПИСАНИЕ КОНЕЧНОЗОННЫХ ПОТЕНЦИАЛОВ
}

\author{
А. Л. Крылов, Г. А. Эль
}

Вводится понятие стохастической солитонной решетки, как случайного процесса, реализациями которого являются конечнозонные потенциалы уравнения Шрёдингера. Изучается эволюция этого процесса в соответствии с уравнением $\mathrm{KдВ}$

Конечнозонные потенциалы уравнения Шрёдингера являются почти периодическими функциями [1], [2] и обладают поэтому естественной стохастической структурой [3]. Для $g$-зонного потенциала (эта структура) задается равномерным распределением на $g$-мерном торе.

1. Напомним, что солитонными решетками [2] называют (квази)периодические решения нелинейных уравнений, в частности, $g$-зонные потенциалы уравнения Шрёдингера, выражающиеся через тета-функцию Якоби на гиперэллиптической поверхности рода $g: \mu^{2}=\prod_{j=1}^{2 g+1}\left(\lambda-r_{j}\right)$,

(1) $u_{g}(x ; \mathbf{r})=C(\mathbf{r})-2 \partial_{x x}^{2} \ln \Theta(x \mid \mathbf{r})=u_{g}(\mathbf{y}(x) \mid \mathbf{r}), \quad y_{j}(x)=k_{j} x+f_{j}, \quad f_{j}(\bmod 2 \pi)$.

Здесь $y_{j}$ - вещественные фазы, $k_{j}$ - волновые числа, $f_{j}$ - начальные угловые фазы. Функция $u_{g}(x \mid \mathbf{r}) g$-квазипериодична по $x$ и задана рядом

$$
u_{g}(x \mid \mathbf{r})=\sum_{j} c_{j} e^{i\left(l_{j} x+h_{j}\right)},
$$

где $c_{j}, l_{j}, h_{j}$ - действительные числа и $-\pi<h_{j} \leqslant \pi$.

Эволюция начальных данных (1) согласно уравнению КдВ описывается линейным сдвигом на якобиане

$$
u_{g}(x, t \mid \mathbf{r})=u_{g}\left(y_{1}(x, t), \ldots, y_{g}(x, t) \mid \mathbf{r}\right), \quad y_{j}(x, t)=k_{j} x+\omega_{j} t+f_{j}
$$

Солитонная решетка (2) называется модулированной, если $\mathbf{r}=\mathbf{r}(\varepsilon x), \varepsilon \ll 1$. Задача Коши для уравнения $\mathrm{K}$ дВ

$$
u_{t}-6 u u_{x}+u_{x x x}=0, \quad u(x, 0)=u_{g}(x \mid \mathbf{r}(\varepsilon x))
$$

имеет решение $u(x, t)=u_{g}(x, t \mid \mathbf{r}(\varepsilon x, \varepsilon t))$, где $u_{g}(x, t \mid \mathbf{r})$ задается формулами (3), а эволюция параметров $\mathbf{r}(X, T), X=\varepsilon x, T=\varepsilon t-$ уравнениями Уизема [2], [4]

$$
\partial_{T} r_{j}+V_{j}(\mathbf{r}) \partial_{X} r_{j}=0, \quad r_{j}(X, 0)=r_{j}(X), \quad j=1, \ldots, 2 g+1 .
$$

2. ОПРеДЕЛЕнИЕ. Стохастической солитонной решеткой (CСР) называется (случайный) процесс $\nu_{g}(x)$, порождаемый конечнозонным потенциалом (1) и заданный формулой $\nu_{g}(x \mid \mathbf{r})=u_{g}\left(\ldots k_{j} x+\phi_{j} \ldots \mid \mathbf{r}\right)$, где $\phi_{j}-$ независимые случайные величины, равномерно распределенные на $(-\pi, \pi]$. Очевидно, процесс $\nu_{g}(x \mid \mathbf{r})$ - эргодический. Разложение Стоуна-Колмогорова для $\nu_{g}(x)$ получается непосредственно из (2) заменой $h_{j} \rightarrow \theta_{j}$, где $\theta_{j}-$ равномерные на $(-\pi, \pi]$ некоррелированные распределения.

Теорема 1. Рассмотрим КдВ (4) как уравнение, описывающее $t$-эволющию однородного процесса $u(x, t)$, с начальными данными $u(x, 0)=\nu_{g}(x \mid \mathbf{r})$. Тогда $u(x, t)=\nu_{g}(x \mid \mathbf{r})$, т.е. ССР $\nu_{g}(x \mid \mathbf{r})$ являются стационарными точками для КдВ-эволючии.

Модулированная ССР $\nu_{g}(x \mid \mathbf{r}(\varepsilon x))$ задается формулами (1), в которых $\mathbf{r}=\mathbf{r}(\varepsilon x)$.

Работа выполнена при финансовой поддержке Civilian Research and Development Foundation for the Former Soviet Union and USA (CRDF) (грант № RM1-145). 
ТеОРема 2. Эволющия модулированной ССР задается формулой

$$
\nu_{g}(x, t \mid \mathbf{r}(X, T))=u_{g}\left(\ldots k_{j} x+\omega_{j} t+\phi_{j}, \ldots \mid \mathbf{r}(X, T)\right),
$$

где $\mathbf{r}(X, T)$ определяется уравнениями Уизема (5).

3. Прямые вычисления с учетом эргодичности дают для двух первых моментов величины $\tilde{\nu}_{g}(x \mid \mathbf{r})=\nu_{g}(x \mid \mathbf{r})-C(\mathbf{r})$ простые формулы (в некоторой естественной нормировке) $:^{1}$ :

$$
\left\langle\tilde{\nu}_{g}(x \mid \mathbf{r})\right\rangle=\frac{1}{4 \pi i}(\mathbf{k}, B \mathbf{k}), \quad\left\langle\tilde{\nu}_{g}^{2}(x \mid \mathbf{r})\right\rangle=-\frac{1}{4 \pi i}(\boldsymbol{\omega}, B \mathbf{k}) .
$$

4. Для уравнения Шрёдингера с почти периодическим потенциалом $q(x)$ в [5] введено число вращения $\alpha(\lambda)$, фактически совпадающее с плотностью состояний $\rho(\lambda)=\frac{1}{\pi} \alpha(\lambda)$. Для конечнозонного $q(x)=u_{g}(x \mid \mathbf{r})$ имеет место соотношение $\alpha(\lambda)=d p(\lambda) / d \lambda$, где $d p(\lambda)$ - квазиимпульс. Интегральная плотность состояний $N(\lambda)$ и полная интегральная плотность состояний $\nu=N(\infty)$ вводятся равенствами

$$
N(\lambda) \equiv \int_{-\infty}^{\lambda} \rho\left(\lambda^{\prime}\right) d \lambda^{\prime}, \quad \nu \equiv N(\infty)=\frac{1}{\pi} \int_{-\infty}^{\infty} d p(\lambda)=\frac{1}{2 \pi} \sum_{j=1}^{g} k_{j} .
$$

Особенно важный смысл имеют эти величины для изучения асимптотического случая $g \gg 1$. При $k_{j}=O\left(g^{-1}\right)$ равномерное распределение угловых фаз $\phi_{j}$ на $T^{g}$ переходит в пуассоновское распределение линейных фаз $l_{j} \equiv \phi_{j} / k_{j}$ на $\mathbb{R}$ со средним значением $\nu$. При $\nu \ll 1$ линейные фазы $l_{j}$ дают асимптотические положения солитонов - этот случай соответствует "солитонной турбулентности" Захарова [6]. Случай большой полной интегральной плотности $\nu \gg 1, k_{j}=o(1)$ отвечает нормальному распределению линейных фаз. ССР является естественным средством описания "предела слабой дисперсии как детерминированного аналога турбулентности" (Лакс, [7]).

Авторы благодарны С. Венакидесу, С. А. Молчанову, С.П. Новикову и М. И. Фрейдлину за полезные обсуждения.

\section{СПИСОК ЛИТЕРАТУРЫ}

[1] Новиков С. П. (ред. ) Теория солитонов. Метод обратной задачи рассеяния. М.: Наука, 1980. [2] Дубровин Б. А., Новиков С. П. // УМН. 1989. Т. 44. №6. С. 29-98. [3] Пастур Л. А., Фиготин А. Л. Случайные и почти периодические самосопряженные операторы. M.: Наука, 1991. [4] Flaschka H., Forest M. G., McLaughlin D. W. // Commun. Pure Appl. Math. 1980. V. 33. P. 739-784. [5] Johnson R., Moser J. // Commun. Math. Phys. 1982. V. 84. P. 403-438. [6] Захаров B. E. // ЖКЭТФ. 1971. T. 60. C. 1012. [7] Lax P. D. // AMS/IP Stud. Adv. Math. 1997. V. 3. P. 53-64.

Институт физики Земли им. О. Ю. Шмидта РАН, Москва;

Институт земного магнетизма, ионосферы

Принято редколлегией и распространения радиоволн РАН, Троицк, Московская обл.

26.01.1999

\footnotetext{
${ }^{1}$ Формула для $\left\langle\tilde{\nu}_{g}\right\rangle$ была указана авторам С. Венакидесом.
} 